\title{
VIE SEXUELLE DES PERSONNES ÂGÉES EN INSTITUTION : CE QU'ELLES EN DISENT
}

\author{
Alexandre Lambelet, Nicole Brzak, Maurice Avramito et Valérie Hugentobler
}

Caisse nationale d'assurance vieillesse | «Gérontologie et société »

2019/3 vol. $41 / \mathrm{n}^{\circ} 160$ | pages 155 à 168

ISSN 0151-0193

ISBN 9782858231225

Article disponible en ligne à l'adresse :

https://www.cairn.info/revue-gerontologie-et-societe-2019-3-page-155.htm

Distribution électronique Cairn.info pour Caisse nationale d'assurance vieillesse.

(C) Caisse nationale d'assurance vieillesse. Tous droits réservés pour tous pays.

La reproduction ou représentation de cet article, notamment par photocopie, n'est autorisée que dans les limites des conditions générales d'utilisation du site ou, le cas échéant, des conditions générales de la licence souscrite par votre établissement. Toute autre reproduction ou représentation, en tout ou partie, sous quelque forme et de quelque manière que ce soit, est interdite sauf accord préalable et écrit de l'éditeur, en dehors des cas prévus par la législation en vigueur en France. Il est précisé que son stockage dans une base de données est également interdit. 


\title{
Vie sexuelle des personnes âgées en institution : ce qu'elles en disent
}

\section{Alexandre LAMBELET}

Professeur associé, HES-SO, Haute école de travail social et de la santé Lausanne (HETSL), Réseau de compétence «Âge, vieillissements et fin de vie » (AVIF)

\section{Nicole BRZAK}

Chargée de recherche, HES-SO, Haute école de travail social et de la santé Lausanne (HETSL), Réseau de compétence «Âge, vieillissements et fin de vie » (AVIF)

\section{Maurice AVRAMITO}

Chargé de recherche, HES-SO, Haute école de travail social et de la santé Lausanne (HETSL), Réseau de compétence «Âge, vieillissements et fin de vie » (AVIF)

\section{Valérie HUGENTOBLER}

Professeure associée, HES-SO, Haute école de travail social et de la santé Lausanne (HETSL), Réseau de compétence «Âge, vieillissements et fin de vie » (AVIF)

Résumé - La manière dont est pensée et vécue la sexualité par les personnes âgées est aujourd'hui encore très peu documentée, a fortiori à propos des personnes qui résident en institution. Et rares sont les études empiriques plaçant au cœur de leurs dispositifs d'enquête la parole et les perceptions des personnes âgées elles-mêmes. Cet article, sur la base d'une enquête qualitative menée auprès de résidents d'établissements de long séjour de Suisse romande, interroge ces éventuels besoins ou envies, de même que l'effet de l'entrée en institution sur l'expression de cette vie affective et sexuelle.

Mots clés - sexualité, grand âge, vieillesse, institution, effets d'âge

\begin{abstract}
The sex lives of elderly people in nursing homes: What residents have to say about it

The way in which elderly people think about and experience sexuality is still very poorly documented, especially in terms of elderly people in nursing homes. Few empirical studies place the voices and perceptions of elderly people at the heart of their research design. This article, based on a qualitative survey of residents in long-term care facilities in French-speaking Switzerland, examines these needs or desires, as well as the effect of institutionalization on the expression of this emotional and sexual life.
\end{abstract}

Keywords - sexuality, old age, aging, institution, effects of age 


\section{Une thématique peu documentée}

La manière dont est pensée et vécue la sexualité par les personnes âgées, qu'elles vivent ou non en institution de long séjour, est très peu présente dans la littérature. Les enquêtes générales sur la sexualité ne la prennent, le plus souvent, pas en compte. L'enquête de Nathalie Bajos et Michel Bozon (2012) ne concerne pas les individus au-delà de 69 ans et la situation est similaire en Angleterre, comme le montrent Merryn Gott et Sharron Hinchliff (2003, p. 1618). Quelques études, essentiellement quantitatives, se sont intéressées à la sexualité après 50 ans, par exemple en Suisse (Bucher, Hornung et Buddeberg, 2003) et en Allemagne (Brähler et Berberich, 2009 ; Schultz-Zehden, 2004), aux États-Unis (Bretschneider et McCoy, 1988) ou en Australie (Bauer et al., 2013). Outre le fait que les enquêtes récentes sont rares, la proportion de personnes très âgées interviewées reste relativement marginale et les personnes vivant en institution en sont absentes, à l'exception notable de celle de Judy Bretschneider et Norma McCoy (1988). L'essentiel de la littérature sur la sexualité des personnes très âgées et en institution de long séjour propose avant tout un questionnement éthique ou philosophique (par exemple, dans la littérature francophone : Colson, 2007 ; Ribes, 2009). Ou alors, elle se concentre sur la perception qu'en ont les professionnels, ainsi que sur leurs pratiques en lien avec la sexualité et le corps des résidents (Aizenberg, Weizman et Barak, 2002 ; Bauer, 1999 ; Thibaud et Hannicote, 2007), plutôt que sur la perception qu'en ont les personnes âgées elles-mêmes.

En même temps, et malgré le peu de données à disposition, nombre d'auteurs encouragent à remettre en cause le mythe selon lequel les personnes âgées seraient dépourvues d'envies sexuelles (Lindau et Gavrilova, 2010), et ce également pour les personnes qui résident en institutions et/ou souffrent de troubles cognitifs (Bauer et al., 2013). Pour Amandine Thibaud et Caroline Hannicote, bien qu'elles semblent emprunter "des voies spécifiques, en s'orientant sur la relation affective plus que sur l'acte lui-même", les expressions sexuelles des sujets âgés perdurent "tout en conservant la même force pulsionnelle ", et ce "malgré le contexte institutionnel » (2007, p. 126). Il s'agirait donc d'aller à l'encontre de représentations trop souvent déficitaires de la vie sexuelle ou d'une vieillesse injustement taxée d'asexuée.

Cette recherche ${ }^{1}$, dans une démarche inductive et compréhensive, interroge ainsi la manière dont les personnes âgées qui vivent en institution, mais aussi celles qui pourraient être amenées à y vivre dans un futur proche, parlent de leur vie affective et sexuelle. Expriment-elles une vie affective et sexuelle actuellement, et comment? Que mettent-elles sous ces termes ? Comment articulent-elles leurs représentations et éventuelles attentes actuelles à leur biographie affective, conjugale et sexuelle antérieure ? Et dans une réflexion plus large sur les processus de socialisation : quelles sont les éventuelles évolutions ressenties ou vécues en termes de vie sexuelle et affective imputables à l'entrée en institution?

\footnotetext{
1 Cette recherche a été cofinancée par la Fondation Leenaards dans le cadre de son appel à projet de recherche 2017 « Qualité de vie $65+»$.
} 
Il ne s'agit pas de faire la promotion de la sexualité, mais bien d'interroger l'expression des attentes et du vécu en termes de vie affective et sexuelle des personnes âgées. En effet, faire la part des choses entre les discours sur la sexualité des personnes âgées et la manière dont ces dernières la perçoivent réellement paraît aujourd'hui parfois bien difficile, tant les discours médiatiques sont emprunts d'une forte valorisation de la sexualité, celle-ci participant d'un vieillissement réussi, constituant un indicateur de bien-être et un révélateur d'une bonne santé physique et psychique (Katz et Marshall, 2003). De plus, si les désirs ou attentes des personnes âgées quant à leur vie affective et sexuelle restent largement méconnus, il en va de même de la force socialisatrice des institutions. Du point de vue des résidents, les institutions permettent-elles, voire favorisent-elles l'expression de leurs désirs en termes de vie affective et sexuelle ou, au contraire, rendent-elles de pareilles expressions difficiles, voire impossibles ? Les possibles changements dans l'expression de tels désirs ou attentes sont-ils le résultat d'effets d'âge (i.e. de santé, de veuvage, etc.) et de génération (i.e. représentations de ce qui est acceptable ou non après un veuvage), ou sont-ils également le fait de contextes (ici institutionnels)?

Les institutions de long séjour sont des lieux paradoxaux avec une confusion des espaces privés et publics ; la volonté de protéger les espaces personnels et la nécessité de les enfreindre quotidiennement pour la réalisation de gestes techniques de soin côtoient le souhait de recréer un chez-soi pour les résidents quand bien même il s'y joue des relations asymétriques fortes entre professionnels et résidents (Ribes, 2011).

Les institutions sont alors très souvent décrites comme empêchant la pleine expression ou le plein respect de l'intimité et de la vie privée, dont la sexualité et les relations affectives sont parties intégrantes, comme lorsque Thierry Darnaud explique "l'impossibilité de l'intime dans les institutions gériatriques " (2007, p. 92) ou que Michael Bauer, parlant des résidents en institutions de long séjour, écrit que "leur seule intimité est entre leurs draps" (1999, p. 37). Les raisons en seraient diverses, que ce soit tantôt le fait de logiques institutionnelles (Lambelet, Pichonnaz et Hugentobler, 2017), des exigences de la vie en communauté ou de "mécanismes désexualisants " (Ribes, 2012, p. 162) liés aux attitudes négatives des professionnels et de leur manque de formation (Bauer et al., 2013). Ainsi, jusqu'à peu, la littérature consacrée aux expressions affectives et sexuelles en institution psychogériatrique se limitait très largement au seul questionnement sur les comportements inappropriés (Tucker, 2010).

À l'inverse, si l'institution peut entraver l'expression d'une sexualité, elle pourrait également jouer un rôle la facilitant. Si la vie communautaire restreint certaines libertés, n'ouvre-t-elle pas tout autant des opportunités ? Ainsi, Gérard Ribes (2009), par ailleurs très critique vis-à-vis des institutions, conclut son ouvrage Sexualité et vieillissement en illustrant la possibilité d'une sexualité épanouie à travers l'exemple d'un couple qui s'est rencontré dans une résidence autonomie. L'institution peut être un facteur de privation d'intimité et de liberté, mais elle peut aussi être un lieu protecteur (Grassi et Titus, 2008) permettant des rencontres et des formes de sociabilités que la vie à domicile, parfois synonyme d'isolement, ne permet plus. 
Il ne s'agit ainsi pas de postuler a priori que les institutions empêchent la pleine expression d'une vie affective ou sexuelle en leur sein, mais toute institution étant instance de socialisation secondaire, il faut également se demander si les propos recueillis auprès des personnes âgées en institution sont modelés par les discours et le cadre institutionnels. Dès lors, il convient de comparer les réponses de personnes âgées dans des établissements de long séjour à celles d'un groupe caractérisable comme très proche en termes d'âge et de vulnérabilité mais dont les membres ne résident pas dans ces établissements. Les entretiens menés auprès de personnes âgées fréquentant des Centres d'accueil temporaire (CAT) ${ }^{2}$ à la journée doivent permettre l'observation d'éventuels effets de socialisation propres aux Établissements médico-sociaux (EMS) ${ }^{3}$. Comparer les propos de personnes âgées se rendant dans des CAT avec ceux de personnes résidant en EMS doit nous permettre d'identifier si l'entrée en institution affecte la manière dont les personnes expriment leur vie affective et sexuelle et nous renseigner sur les éventuelles entraves ressenties par ces personnes dans l'expression de ces dimensions de leur existence.

\section{Méthodologie et données}

\section{Donner la parole aux personnes âgées en perte d'autonomie}

Nous avons collecté les propos de personnes âgées résidant dans le canton de Vaud (Suisse), réparties dans deux groupes de populations différents. Ainsi, 15 résidents d'EMS et 14 usagers de CAT ont été interrogés.

Parce que nous partons du postulat initial que la manière dont ces personnes appréhendent leur situation actuelle peut se comprendre à l'aune de leur histoire de vie affective préalable, nos questions ont porté dans un premier temps sur leur vie affective et sexuelle antérieure à leur entrée en institution. Les entretiens ont ensuite abordé la vie affective et sexuelle présente (ou absente) des personnes rencontrées, les potentiels désirs ou attentes qu'elles peuvent exprimer quant à ces dimensions de leur vie et les potentielles barrières qu'elles ressentent dans la réalisation de ces dimensions de leur existence. L'usage d'entretiens semi-structurés sur le mode d'une conversation informelle nous est apparu particulièrement approprié pour aborder ces sujets sensibles, puisqu'ils permettent une grande flexibilité et offrent également des "portes de sortie " aux enquêtés 4 .

En termes de dispositif d'enquête, nous avons sollicité quatre institutions proposant tant du long séjour (EMS) que de l'accueil temporaire (CAT). De concert avec les responsables de ces institutions, et avec l'aide des équipes d'animation,

\footnotetext{
2 Les Centres d'accueil temporaire (CAT) permettent d'accueillir en journée, de manière ponctuelle et régulière, des personnes âgées vivant à domicile mais fragilisées par la vieillesse, un handicap ou l'isolement.

3 Les EMS correspondent à ce que sont, en France, les Établissements d'Hébergement pour Personnes Âgées Dépendantes (Ehpad).

4 Notre guide d'entretien a largement profité des expériences antérieures d'autres chercheurs (en particulier : Bauer et al., 2013 ; Job et al., 1982 ; Schlagdenhauffen, 2014).
} 
nous avons proposé une animation ouverte à tous en lien avec cette thématique (des contes coquins narrés par une conteuse professionnelle ou un atelier de chant sur la thématique de l'amour) et à laquelle nous avons participé. Ces moments ont été l'occasion de présenter la recherche aux personnes présentes. Après avoir partagé un repas avec les participants à l'animation afin de créer un lien de confiance, il leur était proposé de réaliser un entretien avec l'un des enquêteurs dans le prolongement de la journée. Ce dispositif a été conduit 10 fois, permettant de trouver chaque fois entre 2 et 4 volontaires. Systématiquement, un document présentant la recherche a été remis, et le consentement de la personne vérifié et documenté 5 .

Ce dispositif, comme tout dispositif, a des incidences sur les résultats possibles de l'enquête :

- Nous avons opté pour des animations ouvertes à tous, sans concertation préalable avec les professionnels quant à un possible intérêt d'interroger telle ou telle personne ${ }^{6}$. Les professionnels ne sont intervenus que dans la co-organisation des activités d'animation. Nous n'avons donc pas rencontré les personnes qui sont connues des professionnels comme ayant des relations sexuelles, ou parlant beaucoup de leur sexualité, mais les personnes qui ont eu envie de faire un entretien parmi celles qui ont participé aux animations.

- Le choix de commencer les entretiens en abordant les événements marquants de la vie affective des individus a peut-être conduit à focaliser l'attention sur une certaine dimension de la vie affective et sexuelle reliée à la conjugalité et à laisser dans l'ombre certaines dimensions moins avouables, tels que les fantasmes.

- Conscients des difficultés méthodologiques que cela ajoutait, et nous situant dans le cadre d'une recherche exploratoire, nous avons fait le choix de ne pas enquêter dans des institutions dédiées à l'accueil de personnes désignées comme présentant des troubles cognitifs. Cependant, cela ne pouvait évidemment pas complètement nous prémunir de rencontrer dans ces institutions certaines personnes âgées présentant tout de même quelques difficultés liées à des troubles cognitifs. Lorsque, dans certains cas (3 entretiens), l'entretien laissait percevoir des doutes quant à la capacité de la personne à se situer dans le temps, dans l'espace ou dans sa généalogie (mélangeant par exemple prénom du conjoint ou des parents ou des enfants, disant tantôt avoir des enfants, tantôt ne pas en avoir), ces entretiens n'ont pas été retenus, car les propos qui y étaient tenus rendaient la production de données comme l'analyse trop incertaines.

\footnotetext{
5 Le dispositif d'enquête a par ailleurs été soumis à la Commission cantonale d'Éthique de la Recherche sur l'être humain (CER-VD).

6 Dans les discussions avec les équipes de professionnels, comme dans les informations transmises aux clients des CAT ou résidents en EMS, il a toujours été clairement précisé qu'aucune information sur la situation sociale ou médicale des personnes ne nous était donnée par les professionnels, tout comme nous ne transmettrions aucune information individuelle aux professionnels sur les personnes rencontrées.
} 
Un dernier élément doit être relevé. La littérature nous invite à appréhender la sexualité bien au-delà de sa dimension génitale pour tenir compte de son aspect polymorphe et multidimensionnel, à se décentrer de l'acte sexuel proprement dit pour intégrer toutes les envies de séduction, de complicité et d'affection. Cependant, et comme on le verra, les personnes interrogées n'ont pas eu cette définition extensive de la sexualité. Pour elles, la vie sexuelle a renvoyé directement aux pratiques sexuelles génitales. Tout ce qui relève des émotions affectives, comme des gestes de tendresse, n'est pas exprimé comme relevant de la sexualité. Nous avons donc fait le choix dans l'analyse de notre matériau et parce que notre enquête vise spécifiquement à rendre compte du point de vue des personnes âgées, de respecter leurs représentations et taxinomies indigènes (sur ce point, voir Olivier de Sardan, 1998). Dès lors, et lorsqu'il sera question de "relations sexuelles ", cela renverra bien à la seule dimension génitale de la sexualité. Ce qui n'empêche pas que l'analyse aborde également les désirs, envies et gestes de séduction, de complicité ou d'affection, mais comme des pratiques distinctes de la sexualité, parce que pensées comme telles par les personnes interrogées.

\section{Le corpus d'entretiens}

Notre corpus d'analyse comprend 29 entretiens : 14 avec des personnes fréquentant des CAT, 15 avec des personnes résidant en EMS. Les personnes interviewées sont nées entre 1914 et 1955, avec un âge médian assez proche, que les personnes résident en EMS ou qu'elles fréquentent des CAT : 87 ans pour les premières, 85 ans pour les secondes.

D’un point de vue socio-économique, notre corpus est relativement homogène : la plupart des individus ont suivi une formation professionnelle de base et les professions anciennement exercées sont d'abord celles d'employés dans des petites entreprises ou commerces. 25 des 29 interviewés viennent d'un milieu rural. Enfin, quelques parcours migratoires sont présents : 3 individus viennent d'Italie, 2 du Royaume-Uni et 1 de Colombie.

Notre corpus se compose d'une majorité de femmes veuves, dont certaines sont restées seules (13 sur 15) ou se sont remises en couple (2 sur 15).

Sur les 29 personnes interrogées, 18 personnes ne déclarent qu'une seule relation significative : leur conjoint, qu'elles ont rencontré jeunes et avec lequel elles ont mené l'entier de leur existence. Parmi les 11 autres, 5 personnes déclarent avoir construit des nouvelles relations de couple durables, avec ou sans remariage, après un premier mariage. Parmi elles, on dénombre 3 divorces. En outre, 3 personnes ne se sont jamais mariées.

Notre corpus comprend 10 personnes qui sont dans une relation de couple. Parmi les personnes fréquentant des CAT, 2 personnes ont des conjoints placés en EMS et 4 vivent ensemble à domicile. En EMS, parmi les conjoints de nos 4 interviewés en couple, 3 vivent à l'extérieur de l'institution et un couple y réside ensemble.

En sus de ces 10 couples actuels, 3 autres personnes sont entrées en couple à l'EMS mais leur conjoint est décédé depuis. Toutes les personnes rencontrées qui sont entrées en couple à l'EMS ont emménagé dans des chambres doubles. 
Tableau 1 - Récapitulatif des personnes interviewées

\begin{tabular}{|c|c|c|c|c|c|c|c|c|c|}
\hline & Femmes & Hommes & Total & $\begin{array}{c}\text { Âge } \\
\text { médian }\end{array}$ & $\begin{array}{c}\text { Veuf ou } \\
\text { veuve }\end{array}$ & $\begin{array}{c}\text { En couple } \\
\text { actuellement }\end{array}$ & $\begin{array}{l}\text { Jamais } \\
\text { marié }\end{array}$ & Divorcé & $\begin{array}{l}\text { Entrée en } \\
\text { EMS avec } \\
\text { son époux } \\
\text { ou épouse }\end{array}$ \\
\hline EMS & 12 & 3 & 15 & 87 ans & $\begin{array}{c}10 \\
\left(20^{\lambda}+8+9\right)\end{array}$ & $\begin{array}{c}4 \\
\left(10^{\lambda}+3 ㅇ\right) \\
\end{array}$ & $\begin{array}{c}2 \\
(2+) \\
\end{array}$ & $\begin{array}{c}2 \\
(2+) \\
\end{array}$ & $\begin{array}{c}4 \\
\left(1 \jmath^{\lambda}+3 ㅇ\right) \\
\end{array}$ \\
\hline CAT & 9 & 5 & 14 & 85 ans & $\begin{array}{c}8 \\
\left(10^{\lambda}+7+9\right)\end{array}$ & $\begin{array}{c}6 \\
\left(30^{\lambda}+3 \text { + }\right) \\
\end{array}$ & $\begin{array}{c}1 \\
\left(10^{\lambda}\right)\end{array}$ & $\begin{array}{c}1 \\
\left(10^{\lambda}\right) \\
\end{array}$ & - \\
\hline Total & 21 & 8 & 29 & & 18 & 10 & 3 & 3 & 4 \\
\hline
\end{tabular}

On notera qu'aucune personne n'a rencontré son conjoint actuel dans le CAT ou l'EMS qu'elle fréquente. Cet élément n'est pas anodin quand on sait que des difficultés surviennent souvent pour les professionnels quand des couples se forment dans les institutions et qu'ils s'ajoutent à des relations préexistantes ou qu'ils n'emportent pas l'adhésion des familles. De même, et quand bien même nous avons été soucieux de ne pas donner le sentiment d'une normativité hétérosexuelle à notre recherche, personne n'a relaté d'identité, d'expériences ou d'envies homosexuelles.

Parmi ces 29 personnes interviewées, seules 2 personnes, des femmes, disent avoir encore des relations sexuelles, au-delà de gestes affectifs partagés par la majorité des couples. L'une est en EMS, l'autre en CAT. Toutes deux ont des partenaires plus jeunes, qu'elles ont rencontrés respectivement il y a environ une année et 8 ans.

\section{Analyse}

\section{Quand évoquer la sexualité revient à parler de conjugalité et de fidélités}

Parler de vie affective et sexuelle, qui plus est, dans un entretien semi-structuré qui propose de débuter avec la première rencontre amoureuse revient, pour les personnes rencontrées, à parler de conjugalité. Comme nous l'avons mentionné, pour la grande majorité des individus interviewés (18 sur 29), la vie sentimentale et sexuelle s'articule d'abord à la relation à un conjoint, rencontré et épousé jeune et avec lequel on partage toute son existence. Ils ont souvent rencontré leur conjoint entre 16 et 20 ans, et se sont mariés entre 20 et 25. Le décès du conjoint marque alors une rupture très forte. Certains ont connu le divorce, parfois des remariages ou des remises en ménage, d'autres ont été célibataires toute leur vie mais, dans notre corpus, le modèle " un conjoint pour la vie " est largement dominant.

Dès lors, lorsqu'on les interroge sur leur sexualité, la plupart des personnes interviewées la lient spontanément à leur vie conjugale, l'une ne pouvant pas être dissociée de l'autre. C'est donc le couple qui est au cœur de nos entretiens, avec 
toutes les différentes manières de le vivre et de l'appréhender. Certaines ont pu être passionnément amoureuses :

Qu'est-ce que j'ai aimé cet homme ! Je ne pourrai jamais aimer quelqu'un d'autre [...] je suis tombée amoureuse de cet homme, je l'ai toujours eu dans la peau... (Jacqueline, 85 ans, veuve, CAT)

À l'inverse, on peut regretter de ne jamais avoir vraiment connu l'amour :

On se mariait, même si on n'était pas amoureux avant, c'était mon cas. Ce n'est pas que j'ai fait un coup de foudre. J'ai quand même un mari [...]. Mais, le fait d'affectif, tendresse, ces choses-là, il manquait beaucoup. (Yvette, 76 ans, mariée, CAT)

Cependant, parler du couple et de son conjoint n'est pas toujours aisé. Il ressort de nos entretiens une difficulté à verbaliser et qualifier la relation. Ce sont alors des mots comme "gentil ", "honnête ", "poli " qui reviennent le plus souvent au moment de caractériser le conjoint, qu'il ou elle soit vivant(e) ou décédé(e), comme le montre l'extrait suivant avec Suzanne (83 ans, mariée, EMS) :

- Et votre mari, c'est important qu'il soit séduisant encore pour vous ?

Il est gentil. J'ai un très gentil mari, qui s'est marié tard. Il n'avait pas été marié avant. Et vraiment, je ne peux pas demander un plus gentil mari non plus.

- Par exemple, qu'est-ce qu'il fait de gentil avec vous?

Qu'est-ce que vous voulez que je vous dise ? Il est gentil. [...] Mais, je ne peux pas me plaindre de mon mari et il aime bien mes petits-enfants. Pour moi, c'est l'essentiel.

Cette caractérisation très générale du conjoint et de la relation renvoie-t-elle à une forme de pudeur ? À une volonté de préserver une intimité qui fait que l'on n'entre que peu dans les détails avec l'enquêteur dans la situation d'entretien ? Est-ce une difficulté pour ces personnes à se narrer ? Peut-être. Ou alors, on touche justement là à ce qui compte pour la personne, à ce qui est central au moment de parler de soi et de sa manière d'être au monde et au couple. Pour Suzanne, plutôt que la séduction, ce qui compte n'est-ce pas d'abord que son conjoint, qui n'est pas le père de ses enfants, "aime bien [s]es petits-enfants " et lui permette ainsi d'être à l'aise dans son rôle de grand-mère ? Ainsi, et selon Gérard Ribes, la sexualité doit être englobée dans un ensemble plus vaste, et la notion d'" intimité émotionnelle " prévaut chez le couple : "La sexualité ne doit pas être considérée isolément. Elle participe aux différents modes de relations du couple et la satisfaction sexuelle ne peut être détachée des autres plaisirs et satisfactions de l'existence. [...] La sexualité est la concrétisation de la relation au quotidien, elle est un mode relationnel où le "papotage" des corps ne fait que continuer d'autres échanges. C'est cette dimension d'intimité émotionnelle qui prévaut chez le couple âgé " (2009, p. 58).

De manière générale, la vie sexuelle (génitale) est très peu présente. Pour beaucoup, même en couple, celle-ci s'est arrêtée progressivement voilà un certain temps :

Jusqu'à il y a quelques années, oui. Maintenant, effectivement, je n'ai plus les capacités physiques et puis, ça nous est... Enfin [...], ça nous est sorti tout à fait de la tête. Ça ne m'intéresse plus. Et puis, la maladie de ma femme venant encore se greffer là-dessus, il n'y avait plus les désirs comme auparavant. [...] Je n'ai plus d'attirance pour le sexe opposé. Les gestes de tendresse, oui : échanger des sourires, de petits baisers, des caresses, mais plus sur le plan sexuel. (René, 91 ans, marié, CAT) 
Les mécanismes à l'œuvre font écho à d'autres enquêtes. D'abord, on assiste ici à des effets d'âge. Stacy Lindau, Philip Schumm et al., dans une enquête menée entre 2005 et 2006 auprès de 3005 personnes aux États-Unis, écrivent que "la prévalence de l'activité sexuelle décline avec l'âge (73\% parmi les répondants entre 57 et 64 ans, $53 \%$ de ceux entre 65 et 74 ans et $26 \%$ de répondants entre 75 et 85 ans) et que les femmes rapportent significativement moins que les hommes une activité sexuelle" (2007, p. 762). De même, Nathalie Bajos et Michel Bozon (2012, p. 106) montrent comment le manque d'intérêt pour la sexualité progresse régulièrement avec l'âge. Ensuite, on peut relever l'importance d'avoir un partenaire. Tant une étude de l'American Association of Retired Persons (AARP, 2004) que Régis Schlagdenhauffen (2011) ou Thomas Bucher et al. (2003) montrent en effet combien la présence d'un partenaire régulier ou la perte du partenaire (mort, séparation) sont des facteurs déterminants quant au fait de continuer à avoir des relations sexuelles. Les deux seules personnes de notre enquête disant avoir encore des relations sexuelles ont chacune un partenaire régulier. On notera néanmoins que la question du partenaire n'est peut-être pas suffisante. Dans notre cas, d'autres personnes interviewées ont des partenaires et ne font pas l'amour actuellement.

Enfin, Régis Schlagdenhaufen indique un dernier élément susceptible d'expliquer le peu d'activité sexuelle dans le grand âge : la longévité des couples. Il écrit en effet : "La longévité du couple est corrélée à une moindre activité sexuelle. La fréquence des relations sexuelles au sein du couple est donc liée à la durée de la relation" (2011, p. 5). Les deux personnes de notre corpus disant avoir encore des relations sexuelles sont des couples récents au regard des autres.

En outre, après un veuvage, le fait d'envisager une nouvelle vie amoureuse est bien souvent considéré comme impossible. Jacqueline (85 ans, veuve, CAT), lorsqu'on lui demande si elle pourrait retomber amoureuse dit :

[Que ce ne serait] pas digne de moi. J'ai des enfants, des petits-enfants.

Cet état de fait est partagé par la majorité des veuves et veufs que nous avons rencontrés. Sur 18 personnes veuves, seules 3 d'entre elles se sont remises en couple et 3 nous disent éprouver - ou avoir éprouvé - des sentiments amoureux à l'égard de quelqu'un d'autre sans créer de nouvelle relation. Ainsi, pour une minorité des personnes interviewées, l'EMS ou le CAT peut encore être un lieu de rencontre, de complicité ou de drague, selon qu'il s'agisse de dimensions importantes et présentes pour elles. Ainsi, Madeleine (89 ans, veuve, EMS), qui a tout au long de sa vie cultivé des rapports de séduction avec les hommes nous dit :

Il y a en a un surtout, déjà quand je le vois de loin, cela bouillotte. Quand je passe tout près, cela bouillotte. Je fais semblant de rien, mais cela bouillotte. À mon âge, vous vous rendez compte!

- Et c'est agréable?

Oh que si, cela fait plaisir.

De toute évidence, ces résultats sont liés à une multitude de facteurs, dont le parcours de vie, avec ses socialisations multiples, est un élément essentiel. Le temps qui s'est écoulé entre le décès du conjoint et le moment de l'entretien est aussi un élément important. À ces éléments s'ajoute la répartition hommes/femmes au sein des EMS : avec une large majorité de femmes (64\% de femmes dès 80 ans 
en 2016 (OFS, 2017)), la probabilité d'y retrouver un partenaire du sexe opposé est réduite.

Il est difficile de savoir ce qui relève d'effets de génération, d'âge ou plus simplement de processus de socialisation toujours en partie singuliers. On remarque qu'à quelques exceptions près, parler de sexualité avec des résidents en EMS ou des usagers de CAT semble surtout ouvrir sur le récit d'un temps révolu et même oublié. Les propos portent sur le temps qui a passé, qui est passé. Nombreux sont les interviewés qui disent avoir "tourné la page" ou, comme Marcelle (104 ans, veuve, EMS), que "ce qui est passé est passé ". Les propos sont alors un mélange de rupture et de continuité. Rupture avec l'état de santé (cécité, AVC, corps qui vieillit, etc.) et rupture du couple avec le décès du conjoint, mais aussi, continuité des fidélités : fidélité au conjoint et fidélité à ce qui est perçu par ces personnes comme convenable de faire ou de ne pas faire à leur âge.

\section{Et l'institution?}

La conduite d'entretiens, tant auprès de personnes résidant en EMS que de personnes fréquentant des CAT, avait pour but de nous renseigner sur l'impact éventuel de la socialisation institutionnelle liée à l'entrée dans ces institutions. Dans les faits, il n'en est rien.

Effectivement, les récits issus de notre corpus d'entretiens semblent invalider l'une de nos hypothèses de travail selon laquelle les institutions de grand âge empêcheraient la pleine expression ou le plein respect des dimensions sexuelles et affectives des individus qui y vivent. Pour bon nombre d'entre eux, la vie affective renvoie d'abord au souvenir du conjoint décédé et à l'importance des liens familiaux (enfants et petits-enfants) et ne nécessite pas d'interventions spécifiques de leur part ou de la part de l'institution. Étant donné que la sexualité est majoritairement liée à la conjugalité, et au vu du peu de personnes qui déclarent encore avoir des relations sexuelles, ce domaine de leur vie ne pose pas problème en institution. Dans les cas où les personnes ont un partenaire, les rythmes institutionnels semblent davantage " cadrer" les possibles que les contraindre. Les résidents d'EMS s'arrangent pour composer avec ce nouveau cadre faisant ainsi preuve d'agentivité (Bickel et Hugentobler, 2018). Comme le dit Suzanne, 83 ans, en couple avec son deuxième mari, entrés ensemble il y a 3 ans en EMS :

- Et puis de vivre à deux dans une chambre en EMS, est-ce que des fois vous êtes dérangée par les soignants ou je ne sais pas, ceux qui viennent faire le ménage?

- Non, non. Parce que, non, s'ils viennent faire le ménage, nous, on est libre.

- Vous pouvez encore avoir une vie intime et affective avec votre conjoint?

- Oui, on peut avoir une vie intime quand ils sont loin. Comme ça, il n'y a personne qui risque de rentrer.

- Et c'est quand qu'ils sont loin?

- Ils sont loin à 19 h 30 , je crois.

Dans le même ordre d'idées, Colette, 95 ans, veuve, est la seule personne interviewée résidant en EMS qui déclare avoir des relations sexuelles avec son compagnon vivant à domicile. Ces relations ont lieu hors de l'institution, mais lorsqu'ils souhaitent avoir des moments de tendresse dans la chambre de l'EMS, ces derniers font preuve de créativité afin de s'adapter au cadre institutionnel : ouvrant 
simultanément sa porte de chambre et la porte de sa salle de bains, ceci permet de cacher l'intérieur de la chambre tout en ayant une oreille sur ce qui se passe à l'extérieur, elle dit :

Quand ils voient que la porte est ouverte, ils nous entendent parler, et on parle en anglais. Quand ils nous entendent parler ils savent qu'il y a quelqu'un là donc ils nous laissent, ils ne viennent pas. Et je laisse toujours la porte de la salle de bains ouverte donc ils ne voient pas, et ils frappent à la porte et je dis : "Non pas maintenant, je ne veux pas de thé, je vais descendre. "Je fais comme ça et ils ne passent pas la porte de la salle de bains.

Colette justifie ces arrangements par la crainte des commérages des "femmes de ménage " comme elle les nomme.

En même temps, si les professionnels peuvent être perçus comme des personnes dont il faut se méfier, ils peuvent, tout autant, être des objets de désirs pour les résidents et clients. Ainsi, deux des trois personnes qui nous disent éprouver des sentiments amoureux les éprouvent pour des professionnels qui se prêtent volontiers au jeu tout en conservant une distance professionnelle.

Une seule personne, parmi nos interviewés, relatera l'entrée en EMS comme un obstacle à la poursuite de sa vie affective et sexuelle. Thérèse, 82 ans, divorcée, est en couple depuis 8 ans et ne vit pas avec son partenaire. Le fait qu'elle ait été placée récemment sème le trouble dans son couple :

- Et puis si votre copain il vient ici?

- Ah il vient me dire bonjour, oui, il est venu déjà.

- Vous vous voyez où ? À la cafétéria, dans votre chambre?

- C'est égal.

- Il ne pourrait pas rester dormir?

- Faut pas lui demander ça, il dira : "Je suis pas gaga hein! »J'ai qu'à rentrer à la maison. Alors non, là, exclu. C'est comme si j'étais dans un hôpital ici, c'est fini. Non, pour lui, c'est un choc hein.

- C'est un choc?

- Pour lui, oui. De me savoir là, oui.

À suivre nos entretiens, ce qui marque avant tout le discours sur ces institutions et qui distingue les CAT des EMS, c'est la description des formes que prennent les relations sociales dans ces deux lieux. Si les CAT semblent être des lieux de sociabilité importants, les EMS, eux, apparaissent d'abord comme des lieux où l'on échange peu.

Dans les EMS, en effet, on assiste à des discours où les interactions entre résidents sont présentées comme limitées. Claude (85 ans, veuf, EMS) parle de "relations obligatoires". Les gens se (re)-connaissent, échangent parfois quelques paroles ou des sourires mais il ne s'établit pas véritablement de connexions entre les résidents :

Ici je trouve, en EMS, que les gens sont très pudiques. [...] Les gens se disent rien. Moi, je rigole, je souris tout le temps. Ils sont peu, peu, peu communicatifs. (Ginette, 75 ans, veuve, EMS)

Même les personnes en chambre double disent connaître très peu leur voisin de chambre :

Bon, ma compagne de chambre, on s'entend, on s'entend, juste. Elle est vers la fenêtre, c'est elle qui décide d'ouvrir la fenêtre pour la nuit. Moi je suis vers 
la porte, bon ben si j'ai une urgence de pipi ou autre, je n'ai pas besoin de lui demander. Mais on n'a pas de contact. On n'a pas les mêmes idées. Je ne sais même pas ce qu'elle a fait de sa vie. (Odette, 90 ans, veuve, EMS)

Les entretiens menés auprès de personnes fréquentant les CAT montrent un discours quelque peu différent, la finalité de leur venue au CAT étant elle, avant tout, la recherche de contacts. C'est pour lutter contre ces solitudes que des médecins ou des enfants peuvent les encourager à s'y rendre. C'est le cas de Pierre (64 ans, veuf et remarié) qui se rend dans un CAT pour "pouvoir échanger, discuter, communiquer", qui dit avoir "peur de la solitude ". La dimension socialisante de ces lieux semble fonctionner d'autant plus qu'on y connaît des gens d'avant la fréquentation du lieu.

Il y en a beaucoup que je connaissais déjà d'avant. Comme elle, on a fait l'école ménagère ensemble. Vous voyez, elle a mon âge aussi, c'est rigolo. Et on se retrouve là. [...] Comme là, il y a Monsieur et Madame [nom de famille], on se connaît depuis je ne sais pas combien d'années. J'ai du plaisir à venir là. [...] Alors, j'apprécie, comme souvent, on est une grande table au CAT, j'aime bien. Ça, alors, moi, j'aime bien. (Monique, 93 ans, mariée, CAT)

\section{Pour conclure}

Étant entendu que notre corpus est assez particulier - composé de personnes âgées fragilisées qui fréquentent des institutions (CAT ou EMS), qu'il ne compte pas de personnes y ayant rencontré leur conjoint actuel ni de personnes sortant du modèle hétérosexuel -, ce qui caractérise d'abord les résultats de notre recherche, c'est l'homogénéité des parcours de vie des personnes rencontrées. On se marie vers 20 ans, on garde son conjoint pour la vie, et on reste fidèle le plus souvent à celui-ci quand bien même l'autre est décédé. Reprendre une vie sexuelle après ce décès semble alors hors des possibles, l'évocation du "qu'en-dira-t-on " comme le sentiment d'" inconvenance " n'étant que des manières de rappeler des fidélités qu'on se sent le devoir de respecter : fidélité vis-à-vis du conjoint décédé et fidélité vis-à-vis ce qui est considéré comme convenable pour des personnes âgées.

La sexualité, quand elle est pensée comme rapport sexuel, est quasiment absente. Les expressions comme "c'est du passé ", "j'ai tourné la page " sont omniprésentes, comme si le temps de la grande vieillesse n'était plus un temps pour la sexualité, que les préoccupations étaient ailleurs : le regret d'avoir dû quitter sa maison ; de ne plus être en couple pour partager des activités (aller danser ou au restaurant) ; l'absence de complicité avec les équipes soignantes ; ou encore le manque de possibilité de sortir plus souvent dans la nature. Si ces éléments ont été évoqués spontanément au cours des entretiens, c'est certainement que, pour la plupart des personnes interviewées, ce sont ces dimensions socio-affectives qui leur manquent, bien plus qu'une vie sexuelle active.

L'absence de relations sexuelles, ou de désirs de relations sexuelles, contrairement à notre hypothèse de départ, semble alors peu liée à un effet d'institution. Les témoignages recueillis tendent ainsi à laisser penser que, plutôt qu'une forme de répression sexuelle - orchestrée par l'institution vis-à-vis de comportements qui nécessiteraient d'être cadrés et contrôlés -, ce sont des effets d'âge 
(problèmes de santé, veuvage, absence de compagnon ou de compagne) et de génération qui prédominent et qui sont antérieurs à l'entrée en institution.

\section{RÉFÉRENCES}

Aizenberg, D., Weizman, A. et Barak, Y. (2002). Attitudes toward sexuality among nursing home residents. Sexuality and Disability, 20(3), 185-189. doi:10.1023/A:1021445832294

American Association of Retired Persons (AARP). (2004). Sexuality at Midlife and Beyond. Washington, D.C.: AARP. Repéré à : https://assets.aarp.org/rgcenter/general/2004_sexuality.pdf.

Bajos, N. et Bozon, M. (2012). Les transformations de la vie sexuelle après 50 ans : un vieillissement genré. Gérontologie et société, 35(140), 95-108. doi:10.3917/gs.140.0095

Bauer, M. (1999). Their only privacy is between their sheets: Privacy and the sexuality of elderly nursing home residents. Journal of Gerontological Nursing, 25(8), 37-41.

Bauer, M., Fetherstonhaugh, D., Tarzia, L., Nay, R., Wellman, D. et Beattie, E. (2013). I always look under the bed for a man'. Needs and barriers to the expression of sexuality in residential aged care: the views of residents with and without dementia. Psychology \& Sexuality, 4(3), 296-309. doi:10.1080/19419899.2012.713869

Bickel, J. et Hugentobler, V. (2018). Les multiples faces du pouvoir d'agir à l'épreuve du vieillissement. Gérontologie et société, 40(157), 11-23. doi:10.3917/gs1.157.0011

Brähler, E. et Berberich, H. J. (dir.) (2009). Sexualität und Partnerschaft im Alter. Giessen, Allemagne : Psychosozial-Verlag.

Bretschneider, J. et McCoy, N. (1988). Sexual Interest and Behavior in Healthy 80- to 102-Year-Olds. Archives of Sexual Behavior, 17(2), 109-129.

Bucher, T., Hornung, R. et Buddeberg, C. (2003). Sexualität in der zweiten Lebenshällfte. Ergebnisse einer empirischen Untersuchung. Sexualforsch, (16), 249-270. Repéré à : https://vitagate.ch/public/wem_files/Sex_im_Alter/Sexualitaet_2_Lebenshaelfte.pdf.

Colson, M.-H. (2007). Âge et intimité sexuelle. Gérontologie et société, 30(122), 63-83. doi:10.3917/gs.122.0063

Darnaud, T. (2007). L'impossibilité de l'intime dans les institutions gériatriques. Gérontologie et société, 30(122), 91-106. doi:10.3917/gs.122.0091

Gott, M. et Hinchliff, S. (2003). How important is sex in later life? The views of older people. Social Science \& Medicine, 56(8), 1617-1628. doi:10.1016/S0277-9536(02)00180-6

Grassi, C. et Titus, J.-E. (2008). La sexualité et ses enjeux au sein d'une institution accueillant des personnes physiquement handicapées. Reliance, 29(3), 41-45. doi:10.3917/ reli.029.0041

Job, E., Johansen, C., Jones, J. et Spenceley, E. (1982). Interviewing very old people. Australian Journal on Ageing, 1(2), 7-11. doi:10.1111/j.1741-6612.1982.tb00983.x

Katz, S. et Marshall, B. (2003). New sex for old: lifestyle, consumerism and the ethics of aging well. Journal of Aging Studies, 17(1), 3-16. doi:10.1016/S0890-4065(02)00086-5 
Lambelet, A., Pichonnaz, D. et Hugentobler, V. (2017). Les territoires de l'animation en établissements pour personnes âgées : concilier contraintes organisationnelles et souci de qualité de vie des résidents. Lien social et politiques, (79), 93-112. doi:10.7202/1041734ar

Lindau, S. et Gavrilova, N. (2010). Sex, health, and years of sexually active life gained due to good health: evidence from two US population based cross sectional surveys of ageing. British Medical Journal, (340), 1-11. doi:10.1136/bmj.c810

Lindau, S., Schumm, P., Laumann, E., Levinson, W., O'Muircheartaigh, C. et Waite, L. (2007). A Study of Sexuality and Health among Older Adults in the United States. New England Journal of Medicine, (357), 762-774. doi:10.1056/NEJMoa067423

Office fédéral de la statistique (OFS). (2017). Statistiques des institutions médico-sociales, 23 novembre 2017. Repéré à : https://www.bfs.admin.ch.

Olivier de Sardan, J.-P. (1998). Émique. L'Homme, 147, 151-166. doi:10.3406/ hom.1998.370510

Ribes, G. (2009). Sexualité et vieillissement. Lyon, France : Chronique Sociale.

Ribes, G. (2011). L'âgé, I'intimité et l'institution. Dans P. Pitaud (dir.), Sexualité, handicaps et vieillissement (pp. 133-142). Toulouse, France : Eres, coll. "Pratiques du champ social ».

Ribes, G. (2012). Regards sur l'intimité du dément. Gérontologie et société, 35(140), 159-169. doi:10.3917/gs.140.0159

Schlagdenhauffen, R. (2011). Rapports à la conjugalité et à la sexualité chez les personnes âgées en Allemagne. Genre, sexualité \& société, 6 [En ligne]. Repéré à : https://journals. openedition.org/gss/2205?lang=en.

Schlagdenhauffen, R. (2014). Parler de sexualité en entretien. Comment rendre publics des propos privés. Hermès, 69(2), 34-37. Repéré à : https://www.cairn.info/revue-hermes-larevue-2014-2-page-34.htm.

Schultz-Zehden, B. (2004). Weibliche Sexualität in der zweiten Lebenshälfte - Ergebnisse einer empirischen Studie an Frauen zwischen 50 und 70 Jahren. Sexuologie, 10(2-3), 85-89.

Thibaud, A. et Hanicotte, C. (2007). Quelles représentations les soignants ont-ils de la sexualité des sujets vieillissants ? Gérontologie et société, 30(122), 125-137. doi:10.3917/ gs.122.0125

Tucker, I. (2010). Management of inappropriate sexual behaviors in dementia: A literature review. International Psychogeriatrics, 22(5), 683-692. doi:10.1017/S1041610210000189

\section{e-mails auteurs : alexandre.lambelet@eesp.ch ; nicole.brzak@eesp.ch ; maurice.avramito@eesp.ch, valerie.hugentobler@eesp.ch}

